\title{
e-Phaïstos
}

e-Phaïstos

Revue d'histoire des techniques / Journal of the history

of technology

V-1 2016 | 2018

Ville et technique

\section{L'apport saint-simonien dans l'établissement colonial en Algérie}

The Saint-Simonian contribution to the colonial establishment in Algeria

\section{Mohammed Rahmoun}

\section{OpenEdition}

Journals

Édition électronique

URL : http://journals.openedition.org/ephaistos/1130

DOI : 10.4000/ephaistos. 1130

ISSN : 2552-0741

Éditeur

IHMC - Institut d'histoire moderne et contemporaine (UMR 8066)

Référence électronique

Mohammed Rahmoun, «L'apport saint-simonien dans l'établissement colonial en Algérie », e-Phaïstos [En ligne], V-1 2016 | 2018, mis en ligne le 18 janvier 2018, consulté le 07 mai 2019. URL : http:// journals.openedition.org/ephaistos/1130 ; DOI : 10.4000/ephaistos.1130

Ce document a été généré automatiquement le 7 mai 2019.

Tous droits réservés 


\title{
L'apport saint-simonien dans l'établissement colonial en Algérie
}

The Saint-Simonian contribution to the colonial establishment in Algeria

\author{
Mohammed Rahmoun
}

\section{Introduction}

La construction de la colonie d'Algérie est un processus long et complexe qui, depuis le débarquement d'Alger en 1830 jusqu'à l'événement du centenaire de la colonisation en 1930, n'a cessé de changer avec les évolutions politiques et militaires de la France. Dans l'optique de la colonisation d'un territoire allant vers son autonomie, plusieurs villes et villages communautaires ont vu le jour en Algérie sous influence des thèses fouriéristes et saint-simoniennes. Le pays était devenu, au XIX

siècle, un théâtre d'expérimentations sociales et urbanistiques ouvert aux théories des socialistes utopiques européens. La dualité entre colons et militaires allait façonner non seulement le paysage politique mais l'ensemble du paysage culturel et urbanistique de

l'Algérie.

En France, l'histoire intellectuelle du XIX ${ }^{\mathrm{e}}$

siècle est marquée par un foisonnement de doctrines politiques et sociales, constitutives de ce que Karl Marx et Friedrich Engels ont appelé le « socialisme utopique », initiées par Saint-Simon, Charles Fourier, Étienne Cabet et Philippe Buchez pour citer les plus connus. Parmi ces doctrines, seul le fouriérisme et le saint-simonisme s'imposèrent durablement en Algérie. Il s'agit de deux mouvements de pensée diffus qui cherchent à expliciter et orienter les relations naturelles des hommes en développant des modèles théoriques

d'établissement humain et d'organisation du travail. L'apport des doctrines saintsimoniennes en particulier n'a pas été que théorique. La colonisation de l'Algérie en 1830 leur a offert l'opportunité de fonder des sociétés et des organisations de travail nouvelles. L'Algérie, écrivait Enfantin, est « le lieu d'essai convenable pour cette œuvre 1. 》 


\section{L'apport scientifique et technique des saint- simoniens en Algérie}

L'apport de Saint-Simon ${ }^{2}$

(1760-1825) au mouvement qui porte son nom était essentiellement théorique. Ce n'est qu'après la mort du fondateur en 1825 et la reprise de son œuvre par ses disciples, Prosper-Barthélemy Enfantin, Victor Considérant et Ismaël Urbain, que le mouvement passe définitivement à l'action. Dès 1830, les saint-simoniens prennent débat dans les discussions sur l'avenir de la colonie d'Algérie et publient de nombreux articles dans des revues comme

Le Journal des Débats, La Revue d'Orient, L'Algérie Courrier d'Afrique, d'Orient et de la Méditerranée, etc.

Mais ce n'est qu'avec la création de la Société historique algérienne et saRevue africaine

en 1856, que le mouvement saint-simonien inaugure son entreprise scientifique et technique en Algérie.

Issue de la Commission scientifique de l'Algérie qui avait été fondée en 1837, une équipe de chercheurs composée d'Enfantin et d'autres saint-simoniens rassembla entre 1840 et 1842 un important corpus scientifique et artistique, publié entre 1844 et 1867 sous le titre

Exploration scientifique de l'Algérie

. Cette œuvre d'une grande qualité, devint a posteriori une référence incontournable. Cette conscience savante pour les richesses historiques et culturelles de l'Algérie fit des saint-simoniens des défenseurs du patrimoine culturel musulman. C'est sous la pression de ce courant arabophile et hostile aux convoitises des colons, que le maréchal Bugeaud prit des mesures conservatoires avec la circulaire du 25 mars 1844 visant la conservation des monuments historiques et des restes d'antiquité, et celle du 26 août 1845 sur la collecte d'objets archéologiques pour le Musée algérien de Paris.

Les saint-simoniens se lancèrent dans des entreprises aussi variées que novatrices pour leur époque. Ils contribuent énormément au développement du chemin de fer et du transport maritime en Algérie. Ils s'intéressent aussi à l'exploitation minière et au 5 développement de l'industrie agricole. Comme pour les

Explorations scientifique de l'Algérie, les saint-simoniens soutinrent le projet pour l' Exploration des richesses minérales de l'Algérie

. Ainsi, Henri Fournel, ingénieur des mines et fervent militant saint-simonien, se vit chargé dès 1842 de l'étude du sous-sol algérien.

« Le Gouvernement a le désir de faire en Algérie des explorations géologiques approfondies, pour constater, aussi exactement que possible, les richesses minéralogiques que le sol du pays peut renfermer; et, dans ce but, M. le ministre de la guerre me prie de

lui désigner un ingénieur des mines capable de bien remplir cette importante mission 3. 
L'étude démontra que le sol algérien regorgeait de variétés de roches et de minerais comme le fer, le tungstène, l'antimoine, le cuivre, le zinc, le plomb, le soufre, le kaolin, etc. et que les plus grands gisements métallifères se trouvaient dans les monts de l'Atlas

Tellien

. Ces explorations ramenèrent Fournel dans la partie nord-est de l'Algérie où il note l'existence d'une « montagne entière, le Mokta-El-Hadid ${ }^{5}$

, qui sort du milieu des gneiss et ne présente littéralement depuis le pied jusqu'au sommet, [...] qu'une masse de fer oxydulé pur, sans mélange d'aucune roche

. » C'est dans cette partie du pays qu'apparurent les toutes premières concessions minières et avec elles les premières établissements humains industriels en Algérie.

La plupart de ces concessions, Mokta-el-Hadid, Ain-Mokra et Béni-Saf en particulier, étaient proches de la Méditerranée et entendaient exporter leur minerai directement via

la mer, d'où un certain intérêt des saint-simoniens pour le transport maritime. Il en fut ainsi de Victor Poirel. Cet ingénieur et architecte saint-simonien formé à l'École des Ponts-et-Chaussées édifia à Alger, entre 1842 et 1846, le premier port à blocs artificiels. Ce procédé innovant qui consistait à immerger des blocs de béton et à les agencer pour servir d'assises aux jetées, devint mondialement célèbre et fut généralisé à tous les ports d'Algérie.

\section{L'association chez les saint-simoniens}

Selon Saint-Simon, la société française du début du XIX ${ }^{e}$

siècle était malade de l'affrontement entre deux systèmes économiques, un système féodal « dominant

${ }^{7}$ » et un système industriel « dominé ${ }^{8}$

». Pour les saint-simoniens, l'avènement d'une nouvelle société égalitaire ne s'opèrerait que par le triomphe de la « classe industrielle » sur la « classe féodale ». Il s'agissait donc de créer une société de mérite fondée sur une morale terrestre, une morale de travail en

lieu et place d'une société fondée sur une morale divinisée, une morale de privilèges.

Cette vision humaniste qui avait pour principe la fraternité universelle trouvait son sens 9 dans ce que Saint-Simon appelait le nouveau christianisme

. «Les hommes, disait-il, doivent se conduire en frères à l'égard des uns des autres, ce principe sublime renferme tout ce qu'il y a de divin dans la religion chrétienne 9."

En Algérie, la brutalité de la conquête militaire menée par le général Bugeaud poussa Enfantin à publier en 1843 un ouvrage en deux volumes sur la colonisation de l'Algérie 10

. Il y développe une vision d'union entre les chrétiens et les musulmans de part et d'autre de la Méditerranée. Dans cette perspective, l'Algérie devenait la tête de pont de ce que les saint-simoniens qualifièrent plus tard de « Royaume Arabe de France ». 
En 1848, l'Algérie était peuplée d'environ 120.000 Européens contre deux millions et demi d'autochtones vivant principalement des produits du sol, de l'agriculture et de l'élevage. Les colons, dont la plupart fuyaient les maladies et la pauvreté en Europe, ambitionnaient de s'enrichir rapidement en Algérie en s'accaparant les meilleures terres indigènes. Une situation fermement dénoncée par les saint-simoniens, notamment par Enfantin qui commanda au juge Marion une étude sur « la constitution de la propriété dans les tribus en Algérie ». L'étude mit en évidence que le régime de propriété n'était pas identique pour tous les algériens, contrairement à ce que prétendaient les « colonistes » 11

. L'état de la propriété dans les villes et leurs environs différait de celui de la campagne, en particulier dans les tribus. Selon le juge Marion, la terre pouvait être occupée, travaillée et détenue par des tribus sur plusieurs générations sans qu'elle ne soit attachée à un propriétaire au sens européen du terme.

«On ne peut [...] non plus assimiler les droits des tribus aux usages dont jouissent certaines communes en France, encore moins aux droits qu'ont ces mêmes communes sur les biens communaux proprement dits; puisqu'elles possèdent ces derniers à titre de propriétaires (animo domini) et que les tribus n'ont aucun droit de ce genre sur le sol qu'elles occupent ${ }^{12} . »$

C'est en se référant aux conclusions de Mario qu'Enfantin abolit définitivement dans ses écrits le principe de l'assimilation politique et administrative de l'Algérie à la France. Il existait trop de différences entre les deux pays dans les mœurs, la législation et le régime de la propriété pour que les lois françaises soient appliquées à la lettre en Algérie. Les saint-simoniens demandèrent donc à ce que soit revue la législation française sur la propriété des sols en Algérie, qui depuis 1830 ne cessait de dépouiller et d'appauvrir les Algériens au profit des colons européens ayant pour seule préoccupation leur enrichissement personnel. Enfantin ira jusqu'à admettre que « la France n'a pas tout à enseigner en Algérie, qu'il y a de belles et bonnes choses dans le gouvernement et

l'administration des tribus $13 »$.

\section{À côté de nous mais non mêlés avec nous}

Pour préserver les intérêts et la culture des tribus autochtones, les saint-simoniens préconisèrent la séparation entre européens et musulmans. Les deux communautés pouvaient être proches sur le plan de l'économie et des échanges commerciaux, mais il fallait maintenir la distance culturelle. La solution absolue d'organisation des « indigènes musulmans

14

", disait Enfantin, est de les maintenir séparés, « c'est-à-dire à coté de nous et non mêlés avec nous 15

». Un point de vue partagé par les militaires, notamment par le général Bugeaud, qui voyait dans la fixation des musulmans à proximité des centres européens un moyen de les contrôler. 
Sur le plan urbanistique, la séparation entre Français et Algériens musulmans fut clairement affichée. Dans les propositions d'aménagement faites par Enfantin, on peut lire que « la population maure devrait être la base de la population des villages indigènes, que l'on établira à coté de nos colonies civile, c'est-à-dire sur la ligne extérieure du fossé

.» Avec une exception faite pour les juifs algériens, « peuple de Dieu, qui habite au milieu de tous les peuples, disait Enfantin, ils pourraient habiter nos villes, et pourraient habiter aussi les villes des musulmans

. » Partout en Algérie on assiste à cette configuration: un centre ville moderne peuplé d'Européens et une périphérie " ghettoisée » peuplée d'Algériens musulmans.

Les cités ouvrières n'échappèrent pas à cette règle. Béni-Saf, cité minière fondée en 1870 par la société Soumah \& Tafna et construite par la Compagnie Mokta-el-Hadid, affiche la même ségrégation spatiale et ce malgré qu'elle ait été édifiée par une compagnie industrielle. La cité fut construite pour loger les ouvriers et leurs familles venus principalement d'Espagne. On y trouve des maisons en coron pour les ouvriers de la mine, de belles demeures pour les ingénieurs et les travailleurs qualifiés mais aussi une église, une mairie, une infirmerie, une école, un marché couvert, une salle des fêtes et divers commerces. Cette ville ne profitait qu'aux ouvriers européens de la mine. Les ouvriers musulmans et leurs familles étaient regroupés dans le quartier de Ghar-elBaroud à environ quatre kilomètres du centre ville

. Pis encore, l'entreprise de Mokta-el-Hadid n'a pas vu la nécessité de construire des logements décents pour ses ouvriers musulmans. Leur quartier se résumait à des « cahutes en terre au flanc du coteau, au milieu d'une forêt de figuiers de Barbarie

.» Quoiqu'elle ne cessa de s'accoître, la population musulmane resta à l'écart de la population européenne, dans des quartiers périphériques, comme à Zenzela et Boukardan, et ceci jusqu'à l'indépendance de l'Algérie en 1962.

\section{Conclusion}

Les Saint-simoniens, dans leur projet de colonisation pour l'Algérie, cherchèrent à associer les Algériens au développement économique de la colonie. Une vision qui trouva un écho favorable chez les industriels miniers en raison de leurs besoins considérables en main-d'œuvre et de leur proximité intellectuelle des saint-simoniens. 
La cité minière de Béni-Saf est un exemple d'association entre Européens et Algériens dans la production minière. Mais, les ouvriers musulmans et européens bien qu'ils se côtoyaient pendant les heures du travail, restaient très éloignés dans la vie sociale. Chaque communauté fut maintenue dans son milieu culturel et religieux. Un état de fait qui se reflète dans le plan de la ville avec une ségrégation spatiale entre un centre ville moderne européen et une périphérie ghetto musulmane. Enfantin, en reléguant la population musulmane aux « travaux de force ou des industries communes 20

", a fini par créer une population européenne « dominante » détentrice de pouvoir et de richesse et une population musulmane « dominée » prolétarienne. Une vision ségrégationniste qui trahit en quelque sorte la philosophie du père fondateur SaintSimon, dont le but était de remplacer les classes féodales par des sociétés industrielles plus égalitaires.

\section{BIBLIOGRAPHIE}

ALMI Saïd, Urbanisme et colonisation : présence française en Algérie, Sprimont, Pierre Mardaga, 2002

Association des Béni-Safiens, Album de Béni-Saf, Paris, Association des Béni-Safiens, 1988

DUVAL Jules, Le Phlanstère en Algérie, Alger, Bourger, 1848

ENFANTIN Barthélémy Prosper, Colonisation de l'Algérie, Paris, Bertrand, 1843

FourNEL Henri, Richesse minérale de l'Algérie. Tome 1, Paris, Imprimerie nationale, 1849

LAMORICIERE and BEDEAU, Projet de colonisation pour les provinces d'Oran et de Constantine, Paris, Imprimerie royale, 1847

OULEBSIR Nabila, Les usages du patrimoine. Monuments, musées et politique coloniale en Algérie, 1830-1930, Paris, Maison des Sciences de l'Homme, 2004

RAHMOUN Mohammed : «Le patrimoine industriel minier en Algérie : genèse et développement urbain de la cité ouvrière de Béni-Saf», in Anne-Françoise Garçon et al. (dir.), L'aluminium et la calebasse Patrimoines techniques, patrimoines de l'industrie en Afrique, Belfort-Montbéliard, Presses de l'UTBM, 2013, p. 167-174.

Rahmoun Mohammed, ROSA Carolina, « Industrial Colonies in Algeria : the Urban History of the Mining Town of Beni-Saf », Conference Proceedings, One the Surface The Heritage of Mines and Minning, Leeds Metropolotan University, 2011.

Renard Jules, Les Étapes d'un petit Algérien dans la province d'Oran, Paris, Hachette, 1888.

ROBINET M., « Impressions de voyage d'études dans l'Afrique », Revue de l'industrie minérale, juin 1931, p.197-214.

THÉPOT André, Histoire du corps des Mines au XIX`esiècle, Paris, Eska, 1998. 
TOMAS François, « Les mines et la région d'Annaba », Revue de Géographie de Lyon, 1970,

p.31-59.

\section{NOTES}

1. Enfantin Barthélémy Prosper,Colonisation de l'Algérie, Paris, Bertrand, 1843, p. 533.

2.

Claude-Henri de Rouvroy, comte de Saint-Simon, né à Paris le 17 octobre 1760, mort le 19 mai 1825, fut un militaire, économiste et philosophe, fondateur du saint-simonisme. Ses idées sur la société industrielle française ont influencé de nombreux philosophes du XIX ${ }^{\mathrm{e}}$ siècle.

3.

Lettre envoyée au mois d'août 1842 par M. Legrand, sous-secrétaire d'État des travaux publics, au nom du duc de Dalmatie, ministre de la guerre, à M. Henri Frournel ingénieur en chef des mines,

in Fournel Henri, La richesse minérale de l'Algérie. Accompagnée d'éclaircissements historiques sur cette partie de l'Afrique

septentrionale

, Paris, Imprimerie nationale, 1849, p. ix.

4.

Cet ensemble montagneux d'Algérie, allongé en bordure littorale s'étire sur environ 1500 kilomètres le long de la côte méditerranéenne, en culminant à 2308 mètres d'altitude.

5.

Mokta-El-Hadid, nom arabe de la montagne, signifie " carrière de fer ». Cela laisse supposer que le site minier était vraisemblablement connu et exploité bien avant l'arrivée des Français.

6. Fournel Henri,op.cit., p. iv.

7.

Il s'agissait des nobles, des prêtres, des rentiers et des militaires qui tiraient leur pouvoir de titres acquis.

8.

Il s'agissait des ouvriers, des artisans, des agriculteurs, des entrepreneurs, des scientifiques, des intellectuels et des artistes qui tiraient leur pouvoir de leurs aptitudes individuelles.

9. Saint-Simon Claude-Henri de, « De l'organisation sociale, fragment d'un ouvrage inédit ", Oeuvres complètes, t. 5, Paris, Anthropos, 1966, p. 115 cité par Almi Saïd,

Urbanisme et colonisation : présence française en Algérie, Sprimont, Mardaga, 2002, p. 9.

10. Enfantin Barthélemy Prosper,op.cit.

11. Le terme désignait les partisans politiques des colons.

12.

Marion Amédée, «Lettre à M. Enfantin sur la constitution de la propriété en Algérie » 1842 cité par Almi Saïd, op.cit., p. 30.

13. Enfantin Barthélemy Prosper,op. cit., p. 148

14.

Avec cette qualification de «musulman », Enfantin exclut les Juifs de la population indigène et leur réserve un destin aux côtés de la population européenne et une égalité civile avec les

Français.

15. Ibidem, p. 481.

16. Ibid., p. 482.

17. Ibid. 
18. Association des Béni-Safiens,Album de Béni-Saf, Paris, 1988, p. 157.

19. Ibidem, p. 296.

20. Barthélemy Prosper Enfantin,op. cit., p. 482.

\section{RÉSUMÉS}

La colonisation de l'Algérie a suscité un engouement intellectuel important chez les socialistes utopiques français du XIX

siècle. Dès 1830, les saint-simoniens prennent débat dans les discussions sur l'avenir de la colonie d'Algérie et publient de nombreux articles dans

Le Journal des Débats, La Revue d'Orient, Revue africaine, L'Algérie Courrier d'Afrique, d'Orient et de la

Méditerranée, etc.

En Algérie, la conception du mouvement est formulée par Enfantin et développées par Urbain. Les saint-simoniens contestent fortement le projet d'assimilation de l'Algérie à la France. Conscients de la réalité culturelle et historique du pays, ils plaident pour une colonisation associant Européens et Algériens. L'apport des saint-simoniens à la colonisation algérienne n'est pas que théorique. Leur doctrine économique et sociale les entraîne dans des entreprises diverses et novatrices. Ils prennent part aux explorations scientifiques et minières de l'Algérie, impulsent le développement du chemin de fer et du transport maritime, influencent la politique impériale grâce à leurs travaux sur les régimes de la propriété des algériens. Il s'agit dans cet article de mettre la lumière sur l'une des manifestations intellectuelles les plus influentes dans la

colonisation algérienne, de comprendre la doctrine saint-simonienne sur la société coloniale algérienne et son retentissement sur les sciences, les techniques et l'urbanisme.

The colonization of Algeria has generated a significant intellectual craze among French utopian socialists of the nineteenth century. Early as 1830, the Saint-Simonians take debate in discussions

They published numerous articles in journals such as on the future of the colony of Algeria.

Le Journal des Débats, La Revue d'Orient, Revue africaine, L'Algérie Courrier d'Afrique, d'Orient et de la Méditerranée, etc.

In Algeria, the design of the movement is made by Enfantin and developed by Urban. The SaintSimonians strongly contest the assimilation of Algeria to France. More aware of the cultural and historical reality of the country, they call for a colonization that combines European and Algerian people. The contribution of the Saint-Simonians to the colony of Algeria was not only theoretical. Their economic and social doctrine leads them in various and innovative enterprises. They take part in scientific and mining exploration in Algeria, they boost the development of railway and

maritime transport, and they influence imperial policy through their work on the ownership regime of Algerians land. This article sheds light on one of the most influential intellectual manifestations upon the Algerian colonization. It tries to understand the Saint-Simon doctrine upon the Algerian colonial society and to see its impact on science, technics and urban planning. 
INDEX

Thèmes : Horizons internationaux

Keywords : History of technology, Algeria, Saint-Simonian, French colonization, urban planning, Beni-saf

Mots-clés : Histoire des techniques, Algérie, saint-simonisme, colonisation française, urbanisme, Béni-saf

\section{AUTEUR}

\section{MOHAMMED RAHMOUN}

Docteur en Histoire des techniques de l'université de Paris-1 Panthéon Sorbonne, diplômé du Master Erasmus-Mundus TPTI (Techniques, patrimoines et territoires de l'industrie) des universités Paris 1, Padoue et Evora, diplômé d'un Magistère en Conservation du patrimoine architectural de l'université de Tlemcen et Architecte d'État, Mohammed RAHMOUN est maîtreassistant au département d'Architecture de l'Université de Tlemcen. Il est chargé de cours "Histoire critique de l'architecture" et responsable de la formation "Master Architecture". Ses recherches portent sur les interactions entre technique, société et architecture (XIXe-XXe siècle). Elles relèvent à la fois de l'histoire des techniques et de l'architecture. L'analyse spatiale des cités ouvrières algériennes accompagnant le développement industriel colonial l'a conduit à s'intéresser aux systèmes des transferts techniques métropole/colonie, aux modes d'établissement urbains coloniaux et à la question de la protection du patrimoine industriel en Algérie. 